\title{
Efectos de la asistencia, densidad de la misma y la capacidad del pabellón en las victorias conseguidas en casa en función de la conferencia en la NBA
}

\author{
Effects of attendance, crowd density and capacity of arena \\ in home advantage according to NBA conference
}

\section{Efeitos da assistência, a mesma densidade e capacidade do pavilhão com as vitórias em casa em termos de conferência na NBA}

\author{
Javier García Rubio ${ }^{1}$ María Cañadas Alonso² y Antonio Antúnez Medina ${ }^{3}$ \\ 1 Instituto de Actividad Física y Salud. Universidad Autonoma de Chile, 2 Facultad de Ciencias del Deporte. Universidad de Murcia \\ y 3 Facultad de Educación. Universidad de Extremadura.
}

\begin{abstract}
Resumen: Los equipos que juegan en casa ganan más del 50\% de los partidos. El estudio de los efectos relacionados con el apoyo del público ha sido poco analizado. El objetivo de este trabajo fue identificar los efectos de la capacidad del pabellón, la asistencia a los partidos y la densidad de la misma en las victorias conseguidas en casa. Se analizaron los resultados por equipo de 7 temporadas de la NBA ( $\mathrm{N}=210)$. Los datos fueron extraídos de la página web especializada Basketball-Reference.com. La asistencia media, la capacidad del pabellón y la densidad de la asistencia y su relación con el número de victorias en casa fueron analizadas. Se utilizó un análisis de regresión para analizar los efectos de estas variables en el número de victorias en casa. Los resultados muestran un efecto estadísticamente significativo de la asistencia media al pabellón en ambas conferencias, y de la capacidad del pabellón en la conferencia oeste. El tamańo del público ya se ha demostrado como uno de los predictores más potentes de las victorias en casa. El aumento de la asistencia al pabellón va a llevar a un aumento del rendimiento de equipo. Los clubes deben promover políticas que hagan más atractivo desplazarse al pabellón por parte de los aficionados, aumentando la asistencia y, por tanto, el número de victorias en casa.

Palabras clave: Público, Baloncesto, Localización partido.

Abstract: Home teams wins over the $50 \%$ of games. Studies that explain the associated factors with crowd, have been poorly studied. The aim of the study was to identify interactive effects of facilities size, game attendance and crowd density in home wins. Data were composed by 7 regular season from NBA, 2007/2008-2012/213. Each team of competition was analyzed $(\mathrm{N}=210)$. Data were collected from specialized website Basketball-Reference.com. Regression analysis were used to predict the number of home
\end{abstract}

victories through interactive effects of facilities size, game attendance and crowd density. Results show a significant effect of game attendance in home victories in all the competition and in the analysis split by conferences. Significant effects of facilities size was found for all competition and west conference. Game attendance has been probed as one of the most important home victories predictor. An increase on game attendance tend to a rise in number of home victories.

Key words: Crowd Size, Basketball, Home Advantage.

Resumo: As equipes que jogam em casa mais de $50 \%$ dos jogos. O estudo dos efeitos relacionados com o apoio do público foi pouco analisado. $\mathrm{O}$ objetivo deste estudo foi identificar os efeitos da capacidade do ginásio, a assistência aos jogos e a densidade da mesma nas vitórias conseguidas em casa. Foram analisados os resultados por equipe de 7 temporadas da NBA $(\mathrm{N}=$ 210). Os dados foram extraídos da página web especializada Basketball-Reference.com. A assistência média, a capacidade do ginásio e a densidade da assistência e sua relação com o número de vitórias em casa foram analisados. Utilizou-se a análise de regressão para analisar os efeitos destas variáveis no número de vitórias em casa. Os resultados mostram um efeito estatisticamente significativo da assistência média ao ginásio em ambas conferências e da capacidade do ginásio na conferência oeste. O tamanho do público foi demonstrado como um dos preditores mais potentes das vitórias em casa. O aumento da assistência ao ginásio leva a um aumento do rendimento da equipe. Os clubes devem promover políticas que façam mais atrativo ir ao ginásio por parte dos torcedores, aumetando a assistência e, por tanto, o número de vitórias em casa.

Palavras chave: Tamanho Público, Basquetebol, Jogar em casa.

\section{Introducción}

La ventaja de jugar en casa se conoce como el hecho de que los equipos que juegan en casa ganan más del 50\% de los partidos (Courneya \& Carron, 1992; Garcia, Saez, Ibañez, Parejo, \& Cañadas, 2009). Los factores que explican este fe- nómeno están asociados a los efectos del público, la actuación de los jueces o árbitros, el desplazamiento, la familiaridad con la instalación, el sentimiento de protección de su propio territorio o territorialidad, las diferentes tácticas que utilizan los equipos locales o visitantes para lograr la victoria y los aspectos psicológicos relacionados, como puede ser adoptar una estrategia más defensiva al pensar que es más fácil perder que ganar cuando juegas como visitante (Pollard, 2008). 
Se ha demostrado que los equipos que juegan en la capital del país consiguen una ventaja de jugar en casa menor que el resto de equipos en la competición (Gómez \& Pollard, 2011). En la N.B.A. los equipos pertenecen, en su mayoría, a grandes áreas metropolitanas, con características similares a capitales del país en Europa. Cruzar varios husos horarios tiene efectos a la hora de jugar como visitante (Goumas, 2014). Sin embargo, este efecto es positivo si se juegan varios partidos en el desplazamiento (Pace \& Carron, 1992). El sistema de juego de la N.B.A., con giras con varios partidos en la conferencia contraria, hace que los factores asociados al desplazamiento disminuyan su importancia. Los efectos relacionados con la asistencia a los partidos por parte de los aficionados juegan un papel importante a la hora de explicar la ventaja de jugar en casa. Pocos trabajos han estudiado los efectos de la cantidad del público o la densidad (relación entre la cantidad de público y la capacidad del pabellón) del mismo (Goumas, 2014). Se han estudiado los efectos que puede tener el público en la ventaja de jugar en casa, pero en algunos casos ha sido de manera indirecta, a través de índices como el ranking FIBA (Pollard \& Gómez, 2014). En otros no han encontrado relación entre el aumento de la ventaja de jugar en casa y el aumento del número de aficionados (Nevill, Newell, \& Gale, 1996). Estos autores estudiaron los efectos en ligas de fútbol con asistencias que iban desde los 4000 hasta los 30000 aficionados. Goumas (2014), por su parte, si encontró una relación positiva entre el aumento del público y la ventaja de jugar en casa. Este efecto del público puede influir en las decisiones que toman los árbitros, pero los resultados no están claros (Boyko, Boyko, \& Boyko, 2007; Johnston, 2008) o hacer que los jugadores visitantes realicen acciones más imprudentes. Por otra parte, la influencia de la densidad del público ha sido estudiada en fútbol, con valores que iban desde el 20\% hasta el 70\% de la capacidad total del estadio, sin encontrar diferencias en la ventaja de jugar en casa (Pollard, 1986). Las victorias en casa dependen de varios factores que interactúan entre sí y la relación y cuantificación de estos efectos no está clara (Pollard, 2006).

En la, N.B.A., se han encontrado valores en torno al 60\% en la ventaja de jugar en casa (Carron, Loughhead, \& Bray, 2005; Trandel \& Maxcy, 2011). Sin embargo, se ha demostrado que en varias ligas europeas la ventaja de jugar en casa es mayor que en la liga americana (Pollard \& Gómez, 2013), debido principalmente al sentimiento de proteccion territorial en paises con una historia de conflictos (Pollard, 2006), como en los países de la antigua Yugoslavia (Pollard \& Gómez, 2013). En la N.B.A. todos los equipos pertenecen a una gran ciudad, normalmente la más representativa de un estado. Los pabellones tienen una gran capacidad (CP) y asistencia media (AM), así como densidad del público (DP). Por todo lo anterior, el objetivo del presente estudio es analizar los efectos interactivos de la CP, la AM y la DP en las victorias conseguidas en casa en la NBA en función de la conferencia y para toda la competición. Se ha especulado que la mayor asistencia a los partidos y la mayor densidad del público tendrán un impacto positivo en las victorias en casa en ambas conferencias.

\section{Método}

El diseño del estudio se clasifica como un diseño empírico con una estrategia asociativa y de tipo predictivo (Ato, López, \& Benavente, 2013). Estos estudios permiten explorar la asociación existente entre las variables.

Muestra. Los datos del estudio fueron obtenidos de la página web oficial de la NBA. Se recogieron los datos de los partidos de la temporada regular de la NBA durante los años 2006-2007 a 2012-2013 (NBA Stats, 2014). Los datos fueron agrupados para cada equipo en cada una de las temporadas $(\mathrm{N}=210)$. Los datos recogidos fueron validados por la página especializada Basketball-Reference.com (Kubatko, 2014). En la N.B.A. el sistema de competición se organiza en función de la proximidad geográfica, dividiendo la liga en dos conferencias (Este y Oeste) y 6 divisiones. El calendario no es regular, jugando contra los de su misma división 4 veces al año, los de su misma conferencia entre 3 y 4 veces y contra los de la otra conferencia 2 veces al año.

Variables. Las variables utilizadas en este trabajo fueron las victorias en casa de cada equipo en cada temporada (VC), la capacidad del pabellón (CP), la asistencia media a los partidos (AM), ésta fue calculada a partir de la asistencia total y dividida por los 41 partidos como local de cada temporada, excepto la temporada del lockout donde solo se jugaron 33 partidos; y la densidad del público (DP), calculada a partir de las dos anteriores.

Análisis estadístico. En primer lugar, el análisis descriptivo de la muestra se utilizó para caracterizar la muestra. Un análisis de regresión múltiple se llevó a cabo para predecir el número de victorias locales a partir de las tres variables predictores. De acuerdo con Field (2009), el análisis de regresión añade a cada variable predictiva un coeficiente que permite, al multiplicarlos por su variable y un término aleatorio, explicar la variable dependiente:

$$
\mathrm{VCi}=\beta 0+\beta 1 \mathrm{XCP}+\beta 2 \mathrm{XAM}+\beta 3 \mathrm{XDP}+\varepsilon \mathrm{i}
$$

Para el análisis la muestra se dividió en función de la conferencia a la que pertenece cada equipo. Los resultados del análisis fueron revisados a través del estadístico de DurbinWatson. Este análisis se utiliza para detectar la presencia de autocorrelación en los residuos del modelo. El software estadístico SPSS 22.0 se utilizó para realizar todos los análisis (SPSS Inc., Chicago, IL, USA). La significación del análisis se estableció en $\mathrm{p}<.05$. 


\section{Resultados}

La tabla 1 muestra los estadísticos básicos en función de la conferencia de juego. Se observa que en la conferencia Oeste se ganan más partidos en casa que en la oeste, igualmente la asistencia media y el porcentaje de asistencia son mayores en esta conferencia.

Tabla 1. Estadísticos descriptivos en función de la conferencia de juego.

\begin{tabular}{lccccccc}
\hline & \multicolumn{2}{c}{ Este y Oeste } & \multicolumn{3}{c}{ Este } & \multicolumn{3}{c}{ Oeste } \\
\hline Victorias en casa & 23.81 & 7.36 & 22.80 & 6.91 & 24.98 & 7.66 \\
Capacidad pabellón & 19215.44 & 1601.07 & 19488.35 & 2061.68 & 18978.85 & 833.90 \\
Asistencia media & 17391.02 & 2243.94 & 17400.78 & 2395.27 & 17434.21 & 2070.17 \\
Porcentaje asistencia & 86.19 & 21.64 & 85.87 & 20.12 & 86.53 & 23.35 \\
\hline
\end{tabular}

Los efectos del análisis de regresión por conferencias se observan en la tabla 2. La relación entre las victorias en casa y las variables independientes son mostrados a través de los valoresbeta (B) y el error estándar (SE B). Este análisis muestra que si alguna de las variables aumenta, la variable dependiente se verá afectada, y si este cambio es positivo o negativo. En este estudio, el análisis explica el $17 \%$ del cambio del modelo en la conferencia este y el $36 \%$ en la conferencia oeste. El análisis muestra que ambas conferencias, si aumenta la asistencia a los partidos, aumenta el número de victorias locales. Por su parte, en la conferencia oeste, si disminuye la capacidad del pabellón, aumentará el número de victorias en casa. Los valores-beta muestran cómo afecta cada predictor en el modelo:

Victorias en casa $=8.30+(-0.001$ Capacidad Pabellón $)+$ (0.002 Asistencia Media) + (-0.000 Densidad del Público) $+\varepsilon \mathrm{i}$

Victorias en casa (este) $=18.43+(-0.000$ Capacidad Pabellón $)+(0.001$ Asistencia Media $)+(-0.000$ Densidad del Público) $+\varepsilon i$

Victorias en casa (oeste) $=35.17+(-0.001$ Capacidad Pabellón $)+(0.003$ Asistencia Media $)+(0.003$ Densidad del Público) $+\varepsilon$ i
Como se ve en las ecuaciones y en la tabla 2, los valores-beta son positivos para la asistencia y negativos para la capacidad del pabellón y la densidad en el Este. En el oeste son positivos para la asistencia y la densidad, mientras que la capacidad del pabellón es negativa. Los valores positivos hacen que las victorias en casa aumenten, mientras que los valores negativos hacen que descienda. En este caso, en el este, y puesto que solo la asistencia media es significativa, los valores indican que si la asistencia media aumenta en una unidad, las victorias en casa aumentan en 0.001 . Es decir, son necesarias mil personas más de media para que el número de victorias en casa durante la temporada aumente en una. En la conferencia oeste, al aumentar la capacidad del pabellón en mil personas, descenderían el número de victorias en una, mientras que aumentaría en tres si aumenta la asistencia media al pabellón en mil personas, al ser ambas significativas. El total de la muestra se comporta de manera parecida, si la capacidad del pabellón aumenta en 1000 personas, las victorias en casa disminuye en una. Por el contrario, si aumenta la asistencia media en mil personas, el número de victorias en casa aumentará en una.

Tabla 2. Predicción de la victoria en casa en la NBA.

\begin{tabular}{|c|c|c|c|c|c|c|c|c|c|}
\hline & \multicolumn{3}{|c|}{ Este y Oeste } & \multicolumn{3}{|c|}{ Este } & \multicolumn{3}{|c|}{ Oeste } \\
\hline & B & SE B & $\beta$ & B & SE B & $\beta$ & B & SE B & $\beta$ \\
\hline Constante & 8.30 & 5.77 & & 18.43 & 11.24 & & 35.17 & 14.32 & \\
\hline Capacidad Pabellón & -.00 & .00 & $*$ & -.00 & .00 & & -.00 & .00 & ** \\
\hline Asistencia Media & .00 & .00 & $* *$ & .00 & .00 & $* *$ & .00 & .00 & ** \\
\hline Densidad Público & .00 & .00 & & -.00 & .00 & & .00 & .00 & \\
\hline $\mathrm{R} 2$ & & & $.23^{* *}$ & & $.17^{* *}$ & & & $.36^{* *}$ & \\
\hline Durbin-Watson & & & 1.7 & & 2.0 & & & 1.5 & \\
\hline Número de observaciones & & & 208 & & 105 & & & 103 & \\
\hline
\end{tabular}

${ }^{*} \mathrm{p}<.05 .{ }^{* *} \mathrm{p}<.001$. 


\section{Discusión}

El objetivo de este estudio fue analizar los efectos interactivos de la capacidad del pabellón, la asistencia media a los partidos y la densidad del público en las victorias conseguidas en casa por los equipos de la NBA. La hipótesis se cumple parcialmente. El aumento de la asistencia a los partidos tiene un efecto significativo en el número de victorias en casa, al igual que la capacidad del pabellón en la conferencia oeste. La densidad del público, sin embargo no tiene efecto significativo en el número de victorias.

La ventaja de jugar en casa en la NBA durante las temporadas 2006-2007 a 2012-2013 fue del 59.6\%. Este porcentaje de victorias locales es menor que en otras ligas europeas (Gómez \& Pollard, 2011). En este estudio, se demostró que los equipos que pertenecían a grandes ciudades, aún sin ser capitales de país, experimentaban menos la ventaja de jugar en casa que el resto de los equipos. Se ha explicado que la territorialidad (García, Ibáńez, Gómez, \& Sampaio, 2014; Neave \& Wolfson, 2003) es el sentimiento de pertenencia a un determinado territorio, y es uno de los factores que más influyen en la ventaja de jugar en casa. En las ciudades más cosmopolitas, con una gran población, este factor tiene menos influencia. Además, la comercialización de la liga hace que los equipos no sean representativos de la comunidad, con cambios de franquicias entre ciudades (Gómez \& Pollard, 2011).

La asistencia media a los pabellones es el único predictor que es significativo en los tres análisis realizados. Uno de los factores más importantes para explicar las victorias en casa es la teoría de la Facilitación Social (García et al., 2014; Gómez, Lorenzo, Ortega, \& Olmedilla, 2007). Esta teoría sostiene que la presencia de espectadores incrementa el rendimiento de tareas más condicionales, relacionadas con situaciones del juego como los tapones o los rebotes defensivos. Los jugadores locales, al sentir el apoyo de su público, son más agresivos en su juego, buscando el contacto físico para intimidar a los visitantes. Esto unido a la hostilidad del público (Sampaio, Ibañez, Gómez, Lorenzo, \& Ortega 2008), lleva a los jugadores visitantes a cometer más errores. El ruido del público también afecta a las decisiones que toman los árbitros (Nevill \& Holder, 1999) e incluso influyen en el rendimiento de los jugadores y los equipos, afectando al porcentaje de tiros libres (Kotecki, 2014). Igualmente, los jugadores locales muestran un aumento en la secreción de testosterona, asociada a un aumento de la competitividad (Neave \& Wolfson, 2003). Los jugadores visitantes, por el contrario, experimentan un incremento en la secreción de cortisol (Mehta \& Josephs, 2010) que puede ser producida por el miedo y el estrés (Lupis, Lerman, \& Wolf, 2014). Esta hormona suprime los efectos de la testosterona. La elevada asistencia a los pabellones de la NBA unido a que los aficionados están a pie de pista, muy cerca de los jugadores (Armatas \& Pollard, 2014), así como las repeticiones en pantallas gigantes donde los jugadores ven sus acciones y sus fallos, pueden ser importantes causantes de ese miedo, aumentando el stress y disminuyendo el rendimiento y la competitividad de los equipos visitantes. Finalmente, las grandes distancias entre equipos, hace que los aficionados tengan que realizar largos desplazamientos para animar a sus equipos cuando juegan como visitantes. Se ha demostrado que en los partidos entre dos equipos de la misma ciudad, donde los aficionados no tienen que desplazarse para animar a su equipo, muestran una menor ventaja de jugar en casa (Seckin \& Pollard, 2008).

La capacidad del pabellón es el otro predictor que tiene un efecto negativo en el número de victorias en casa. En este caso cuanto mayor sea el estadio, más se suaviza el efecto del tamaño del público, al disminuir la densidad. Un gran estadio, si no tiene una gran cantidad de público, puede hacer perder el efecto intimidatorio del mismo. En esta línea, la densidad del público no se asoció con ningún tipo de efecto en el número de victorias en casa, confirmando los resultados de Goumas (2014), donde es más importante la cantidad de público que lo lleno que este el pabellón.

Finalmente, los equipos deben hacer un esfuerzo por llenar sus instalaciones de público, no solamente por el aspecto económico que tiene asociado el aumento de ingresos, sino que van a ayudar a sus jugadores y equipos a lograr más victorias (Kotecki, 2014). En grandes ciudades donde no hay un sentimiento de pertenencia al equipo tan fuerte como en ciudades más pequeñas, o en una nación con un gran compromiso patriótico y sentimiento de unidad como los Estados Unidos, la influencia de la territorialidad puede ser menor que la cantidad de público que apoya al equipo. En el lado contrario, los equipos visitantes tendrán que preparar los partidos de acuerdo a las características del entorno con el que se van a encontrar

\section{Referencias}

1. Armatas, V., \& Pollard, R. (2014). Home advantage in Greek football. European Journal of Sport Science, 14(2), 116-122.

2. Ato, M., López, J. J., \& Benavente, A. (2013). Un sistema de clasificación de los diseños de investigación en psicología. Anales de Psicología, 29(3), 1038-1059.

3. Boyko, R. H., Boyko, A. R., \& Boyko, M. G. (2007). Referee bias con- tributes to home advantage in English Premiership football. Journal of Sports Sciences, 25(11), 1185-1194.

4. Campos, F. A., Pellegrinotti, Í. L., Pasquarelli, B. N., Rabelo, F. N., SantaCruz, R. A., \& Gómez, M.-Á. (2015). Effects of game-location and quality of opposition in futsal league. International Journal of Performance Analysis in Sport, 15(2), 598-607. 
5. Carron, A. V., Loughhead, T. M., \& Bray, S. R. (2005). The home advantage in sport competitions: Courneya and Carron's (1992) conceptual framework a decade later. Journal of Sports Sciences, 23(4), 395-407.

6. Courneya, K. S., \& Carron, A. V. (1992). The home advantage in sport competitions: A literature review. Journal of Sport and Exercise Psychology, 14(1), 28-39.

7. Field, A. P. (2009). Discovering statists using SPSS (and sex and drugs and rock 'n'roll). London: Sage.

8. García, J., Ibáñez, S. J., Gómez, M. A., \& Sampaio, J. (2014). Basketball Game-related statistics discriminating ACB league teams according to game location, game outcome and final score differences. International Journal of Performance Analysis in Sport, 14(2), 443-452.

9. Garcia, J., Saez, J., Ibañez, S. J., Parejo, I., \& Cañadas, M. (2009). Home advantage analysis in ACB league in season 2007-2008. Paper presented at the Revista de psicología del deporte.

10. Gómez, M. A., Lorenzo, A., Ortega, E., \& Olmedilla, A. (2007). Diferencias de los indicadores de rendimiento en baloncesto femenino entre ganadores y perdedores en función de jugar como local o como visitante. Revista de Psicología del Deporte,, 16(1), 41-54.

11. Gómez, M. A., \& Pollard, R. (2011). Reduced home advantage for basketball teams from capital cities in Europe. European Journal of Sport Science, 11(2), 143-148.

12. Goumas, C. (2014). Home advantage in Australian soccer. Journal of Science and Medicine in Sport, 17(1), 119-123.

13. Johnston, R. (2008). On referee bias, crowd size, and home advantage in the English soccer Premiership. Journal of Sports Sciences, 26(6), 563-568.

14. Kotecki, J. (2014). Estimating the Effect of Home Court Advantage on Wins in the NBA. Honors Projects, Paper 124. doi: http://digitalcommons.iwu.edu/econ_honproj/124

15. Kubatko, J. (2014). Basketball-Reference.com- Basketball Statistics and History. . http://www.basketball-reference.com/.

16. Lupis, S. B., Lerman, M., \& Wolf, J. M. (2014). Anger responses to psychosocial stress predict heart rate and cortisol stress responses in men but not women. Psychoneuroendocrinology.
17. Mehta, P. H., \& Josephs, R. A. (2010). Testosterone and cortisol jointly regulate dominance: Evidence for a dual-hormone hypothesis. Hormones and Behavior, 58(5), 898-906.

18. Neave, N., \& Wolfson, S. (2003). Testosterone, territoriality, and the 'home advantage'. Physiology \& Behavior, 78(2), 269-275.

19. Nevill, A. M., \& Holder, R. L. (1999). Home advantage in sport: an overview of studies on the advantage of playing at home. Sports Medicine, 28(4), 221-236.

20. Nevill, A. M., Newell, S. M., \& Gale, S. (1996). Factors associated with home advantage in English and Scottish soccer matches. Journal of Sports Sciences, 14(2), 181-186.

21. Pace, A., \& Carron, A. V. (1992). Travel and the home advantage. Canadian Journal of Sport Sciences.

22. Pollard, R. (1986). Home advantage in soccer: A retrospective analysis. Journal of Sports Sciences, 4(3), 237-248.

23. Pollard, R. (2006). Home Advantage in Soccer: Variations in its Magnitude and a Literature Review of the Inter-related Factors Associated with its Existence. Journal of Sport Behavior, 29(2).

24. Pollard, R. (2008). Home advantage in football: A current review of an unsolved puzzle. The Open Sports Sciences Journal, 1(1), 12-14.

25. Pollard, R., \& Gómez, M.A. (2013). Variations in home advantage in the national basketball leagues of europe. Revista de Psicología del Deporte, 22(1), 263-266.

26. Pollard, R., \& Gómez, M. A. (2014). Components of home advantage in 157 national soccer leagues worldwide. International Journal of Sport and Exercise Psychology, 12(3), 218-233.

27. Sampaio, J., Ibañez, S. J., Gómez, M. A., Lorenzo, A., \& Ortega , E. (2008). Game location influences basketball players performance across playing positions. International Journal of Sport Psychology, 39(3), 43-50

28. Seckin, A., \& Pollard, R. (2008). Home advantage in turkish professional soccer. Perceptual and Motor Skills, 107(1), 51-54.

29. Trandel, G. A., \& Maxcy, J. G. (2011). Adjusting winning-percentage standard deviations and a measure of competitive balance for home advantage. Journal of Quantitative Analysis in Sports, 7(1). 